\title{
A Case of Radical Laparoscopic Central Bisegmentectomy with S3 Subsegmentectomy for Multiple Liver Metastases After Chemotherapy
}

\author{
Mamoru Morimoto, Yoichi Matsuo, Kenta Saito, Ken Tsuboi, Ryo Ogawa, Hiroki Takahashi, Shuji Takiguchi
}

Department of Gastroenterological Surgery,

Nagoya City University Graduate School of Medical Sciences, Japan

\section{ABSTRACT}

Hepatic resection remains the only potentially curative treatment for patients with colorectal liver metastasis (CRLM) (1). Moreover, only $15 \%-20 \%$ of patients with CRLM are suitable for surgical resection (2). Herein wepresent a video case-report of a radical laparoscopic central bisegmentectomy plus S3 subsegmentectomy for multiple liver metastasis after chemotherapy. The patient was a 39-year-old man who was shown to have metachronous multiple liver metastases from sigmoid colon cancer. The size of the tumor in $\$ 3$ was $7 \mathrm{~cm}$, and the size of the tumor in $S 4 / S 5 / S 8$ was $13 \mathrm{~cm}$. At this point, it was judged that there was no indication for surgery, thuschemotherapy was started. ThemFOLFOX+panitumumabwas administered for 8 courses and FOLFILI+panitumumab was administered for 10 courses. The metastatic lesions showed a partial clinical response to the chemotherapy. Because the tumors were limited in $\mathrm{S} 3$ and $\mathrm{S} 4 / 5 / 8$, a radical hepatectomy was thought to bepossible. A CT scan revealed that the tumor in S3was located near the root of G3, while the tumor in S4/S5/S8 was located near the anterior Glissonean pedicle and partially infiltrated G4. To assure resection of the lesion, weperformed a laparoscopic central bisegmentectomy plus S3 subsegmentectomy. Then, volumetry was performed based on VINCENT CT. The volume of the remnant liver was $41 \%(\mathrm{Krem}=0.077)$. We confirmed that the operation couldbe performed safely. Because it was necessary to resect a very large area of the liver in this operation, the degree of difficultywas deemed to be very high.To perform this operation successfully, it wasnecessary to secure a good surgical view and make good use of the magnified view afforded via the laparoscope. Thus, acranial-to-caudal direction hepatectomy was performed. The merit of the procedure is that the amount of bleeding is reduced by resecting the liver parenchyma without reversing the direction of the venous branch (3). Even such a difficult operation can be safely performed by devising a thoughtful approach.

\section{Conflict of interest}

All authors declare that they have no conflicts of interest.

\section{REFERENCES}

1. Adam R, Pascal G, Azoulay D, Tanaka K, Castaing D, Bismuth $\mathrm{H}$. Liver resection for colorectal metastases: the third hepatectomy. Ann Surg 2003; 238: 871-883; Discussion 883-884.

2. Steele G, Ravikumar TS. Resection of hepatic metastases from colorectal cancer. Biologic perspective. Ann Surg 1989; 210: 127-138.

3. Xiao L, Li JW, Zheng SG. Laparoscopic anatomical segmentectomy of liver segments VII and VIII with the hepatic veins exposed from the head side. J Surg Oncol. 2016;114(6):752-756.

\author{
Corresponding author: \\ Yoichi Matsuo, M.D., Ph.D. \\ Department of Gastroenterological \\ Surgery, Nagoya City University \\ Graduate School of Medical Sciences, \\ Kawasumi 1, Mizuho-cho, Mizuhoku, \\ Nagoya 467-8601, Japan \\ Tel: +81-52-853-8226 \\ Fax: +81-52-842-3906 \\ E-mail: matsuo@med.nagoya-cu.ac.jp
}

Received: 20.09 .2019

Accepted: 23.09 .2019 